\title{
3.6 „Auf Facebook erreicht man auch alte Menschen“
}

\section{Dagmar Hirche über ihre Erfahrungen mit Facebook.}

Der Verein Wege aus der Einsamkeit e. V. (WADE) setzt sich für ein würdevolles Leben im Alter ein - und ist damit auf Facebook der große Hit. Über 4.500 Fans verfolgen die Social Media Aktivitäten des kleinen Hamburger Vereins. Warum? Dagmar Hirche, Geschäftsführerin von WADE, über neue Kontakte, das versilberte Netz und tanzende Opas.

Dagmar, warum hast du für deinen Verein eine Facebook-Seite angelegt?

Nachdem ich Gast bei einer Talkshow im SWR war, haben mich zahlreiche Leute angeschrieben und gefragt, wo sie uns denn auf Facebook finden würden. Daraufhin haben wir uns Gedanken gemacht, wie wir das Thema Alter bei Facebook besetzen könnten. Wir haben uns dann ganz bewusst dafür entschieden, das Altern positiv und lebendig darzustellen. Das passt gut zum Medium Facebook, und thematisch war da noch eine Lücke. Wir sind also nicht gleich losgestürmt, sondern haben zunächst eine kleine Strategie entwickelt.

Dein Verein macht Lobbyarbeit für das schöne Altwerden. Doch bei Facebook sind nur wenige Alte unterwegs. Warum seid ihr trotzdem da?

Weil man alte Menschen auch über Facebook erreicht. Nicht nur wächst die Nutzergruppe der Silversurfer auch im Bereich Social Media stark. Hinzu kommt der persönliche Austausch zwischen den Generationen, etwa wenn eine Enkelin ihrer Oma die letzten Facebook-Posts von WADE zeigt und sie gemeinsam lachen und sich über tanzende Opas im Video freuen. Dann ist auch der alte Mensch Teil unserer Facebook-Gemeinschaft. Über solche Berichte freue ich mich sehr. Außerdem ist unsere Facebook-Präsenz immer ein schöner Anlass, um mit Interessierten ins Gespräch zu kommen. Allein schon wegen unseres lustigen Namens. 
Über den Generationenaustausch hinaus - welchen Nutzen hat die Social-Media-Präsenz für euch noch?

Ich nutze Facebook vor allem, um mich mit überregionalen und internationalen Vereinen zu vernetzen, die etwas Ähnliches wie wir machen. Dadurch bekomme ich viele Ideen für unsere Vereinsarbeit und schaue mir manchmal was ab. Zu unserem Wettbewerb „Wir versilbern das Netz" hat mich zum Beispiel eine irische Organisation inspiriert. Außerdem kommen viele Menschen über Facebook auf uns zu. Ich bekomme pro Woche zwischen zehn und dreißig Nachrichten und Anfragen zu unserer Arbeit. Über Facebook erreichen wir Menschen, die nicht unsere Regionalzeitung lesen oder sich nicht unbedingt für das Thema Alter interessieren. Über die Empfehlungen oder Kommentare von Freunden erfahren sie aber von uns.

\section{Wie viel Zeit verbringst du mit der Pflege eurer Social-Media-Präsenzen?}

Den Zeitaufwand darf man nicht unterschätzen. Seit 2014 posten wir etwa dreimal am Tag eine Nachricht. Die Themen zu finden und Ideen zu entwickeln, kostet Zeit. Ich verbringe so zwischen ein und zwei Stunden täglich mit Social Media. Mir macht das aber auch Spaß, und ich habe da eine Routine entwickelt. Für mich ist das ein wichtiger Informationsaustausch. So viel Zeitung könnte ich gar nicht lesen!

\section{Lohnt sich der Aufwand? Sammelt ihr über Social Media Spenden?}

Für uns steht das Spendensammeln in den sozialen Medien nicht im Vordergrund. Wir wollen hier in erster Linie über unsere Arbeit informieren und interessierten Menschen eine zusätzliche Anlaufstelle bieten. Wir haben allerdings die Erfahrung gemacht, dass wir mit großer Social-Media-Reichweite für Unternehmen, die sich engagieren wollen, interessanter sind. Denn wir können vielen Menschen von dem Unternehmensengagement berichten. Nach dem Motto „Tue Gutes und rede darüber“ sind Unternehmen auf uns zugekommen, um uns zu unterstützen. Und darüber twittern wir dann und schreiben einen Facebook-Post.

\section{Hast Du Tipps für Organisationen, die Facebook nutzen wollen?}

Mir begegnen viele Organisationen, die zunächst kritisch sind. Die sagen: „Warum sollen wir da freiwillig etwas von uns preisgeben?" Ich bin aber davon überzeugt, dass wir gemeinnützigen Organisationen tolle Arbeit machen, und ich möchte davon berichten, was wir tun, und dem 
auch ein Gesicht geben. Das ist nicht jedermanns Sache, da muss man in der Organisation gucken, ob es jemanden gibt, der sich das vorstellen kann. Dabei sollte man keine Angst vor Fehlern haben - auch gemeinnützige Organisationen können lernen und Fehler zulassen.

Außerdem geht es in sozialen Medien auch darum, sein Thema zu verbreiten. Und das möglichst mit Bild, Video und in einer klaren, leichten Sprache. Um diese Vielfalt in sozialen Medien darstellen zu können, muss man sich auch für andere Organisationen und Ansätze öffnen. Und das ist in meinen Augen eine Riesenchance.

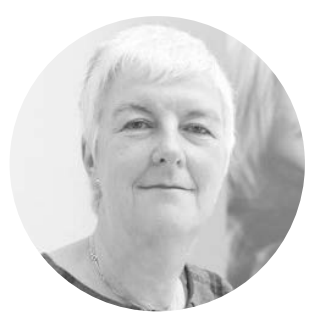

\section{Dagmar Hirche}

Geschäftsführerin

Verein Wege aus der Einsamkeit e. V. (WADE) 